REVUE HYBRIDE DE L'ÉDUCATION

\title{
Consolider ses apprentissages à distance : un virage à $360^{\circ}$
}

\author{
Auteurs \\ Suzie Tardif, doctorante en éducation, Université du Québec à Chicoutimi, \\ Canada, \\ suzie.tardif@uqac.ca \\ Étienne Boulanger, enseignant en arts visuels et numériques, collège \\ d'Alma, Canada, \\ etienne.boulanger@collegealma.ca \\ Martin Maltais, enseignant en anglais langue seconde, collège d'Alma, \\ Canada, \\ martin.maltais@collegealma.ca
}




\section{REVUE HYBRIDE DE L’ÉDUCATION}

\section{Résumé}

Depuis quelques années, deux enseignants-chercheurs du collège d'Alma (Lac-Saint-Jean) ont développé une expertise en pédagogie immersive. Bien que leurs travaux liés à la vidéo $360^{\circ}$ aient débuté avant la pandémie de COVID-19, ils ont suscité énormément d'intérêt auprès de divers intervenants pendant celle-ci. Le contexte, la mise en œuvre et les retombées de cette pratique pédagogique innovante seront dès lors décrits dans cet article.

Mots-clés : pédagogie immersive; réalité étendue; expérience immersive virtuelle; vidéo $360^{\circ}$; enseignement à distance 


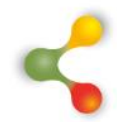

\section{REVUE HYBRIDE DE L'ÉDUCATION}

\section{Mise en contexte}

II ne fait plus de doute que les technologies de l'information et de la communication (TIC) permettent "d'ouvrir les murs de la classe" (Stockless, 2018, p.3) puisqu'elles invitent les étudiants à vivre des occasions d'apprentissage en dehors de celle-ci. Depuis quelques années, notamment au postsecondaire (Harrington et al., 2018 ; Roche et Rolland, 2020), les enseignants se tournent ainsi vers des technologies de réalité étendue, communément appelées XR, pour innover dans leur milieu. Parmi celles-ci se trouvent la réalité virtuelle (RV), la réalité augmentée (RA), la réalité mixte $(\mathrm{RM})$ et la réalité immersive $360^{\circ}$ (ou vidéo $360^{\circ}$ ). Cette dernière demeure de plus en plus exploitée en éducation compte tenu, entre autres, de son coût de production considéré plus abordable (Aguayo, Cochrane et Narayan, 2017). Tel que le résument Roche et Rolland (2020) :

La vidéo $360^{\circ}$ se différencie de la RV car elle permet à l'individu qui la visionne de tourner dans l'espace enregistré, de s'orienter dans l'image, mais pas d'interagir avec elle. La RV, la RA ou la RM reposent sur des reconstructions informatiques d'environnements réels (extrêmement coûteuses financièrement), alors que la vidéo $360^{\circ}$ rend seulement compte d'environnements réels qui ont été filmés avec des technologies spécifiques, qui rendent possible leur exploration active par un utilisateur (p.29).

Son utilisation en enseignement demeure grandement intéressante. En effet, la vidéo $360^{\circ}$ peut, à titre d'exemple, permettre à des étudiants d'avoir accès visuellement à des lieux d'exercice professionnel, en plus d'acquérir ou de consolider des apprentissages liés à leur formation (Roche et Rolland, 2020). C'est ce potentiel pédagogique qui a motivé deux enseignants-chercheurs du collège d'Alma (Lac-Saint-Jean), rencontrés dans le cadre d'une entrevue semi-dirigée, à accompagner leurs collègues dans l'exploitation de cette technologie de réalité étendue.

Martin Maltais et Étienne Boulanger présentent des parcours scolaires et professionnels empreints d'expériences variées. Alors que le premier enseigne en anglais langue seconde, le deuxième fait partie de l'équipe du département d'Arts visuels et numériques de collège d'Alma. Bien que leurs domaines d'enseignement divergent, tous deux ont un intérêt marqué pour la technologie. Ils ont d'ailleurs participé à plusieurs travaux de recherche portant sur cette thématique, plus précisément sur le développement d'outils technologiques disponibles pour les formations techniques au collégial.

En 2017, après une analyse en profondeur des programmes offerts dans leur collège, des éléments à améliorer ont été relevés, entre autres, 


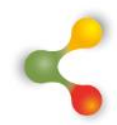

\section{REVUE HYBRIDE DE L'ÉDUCATION}

dans le programme technique en Soins infirmiers. En effet, tel que résumé par un des enseignants-chercheurs consultés :

\begin{abstract}
$\mathrm{Ce}$ programme d'étude présente un contenu particulièrement chargé. Le professeur des soins a un temps limité en classe pour l'enseignement et les démonstrations des méthodes de soins. Ainsi, certaines méthodes ne sont démontrées qu'une seule fois et ensuite pratiquées en laboratoire avant les stages en milieux hospitaliers. Lors de la démonstration en classe des méthodes, les étudiants n'ont pas tous le même point de vue et certains, dans les rangées éloignées, ont un point de vue carrément limité.
\end{abstract}

Ces deux enseignants-chercheurs ont ainsi voulu créer un outil d'accompagnement qui permettrait aux étudiants de se familiariser davantage avec la réalisation de certaines techniques de soin. La vidéo $360^{\circ}$ a dès lors été perçue comme étant un incontournable puisqu'elle pouvait être liée à la réalisation de mises en situation scénarisées et contextualisées qui permettent à un étudiant de consolider ses apprentissages en lien avec son programme de formation.

Ces deux passionnés des TIC se sont ainsi joints à l'équipe enseignante de cette technique pour mettre au point différentes vidéos de réalité immersive. Leurs travaux ont ensuite suscité l'intérêt des autres départements de leur collège (ex.: Techniques policières, Techniques d'intervention en milieu carcéral, etc.) et même trouvé écho de manière plus large. En effet, ils intéressent désormais différents instances et organismes régionaux et provinciaux, depuis la pandémie de COVID-19, considérant les retombées potentielles de cet outil pédagogique sur l'apprentissage. Les prochaines lignes visent à décrire comment mettre en place une telle initiative en exemplifiant la méthode utilisée par les deux enseignants-chercheurs consultés.

\section{Déroulement}

Tel que précisé antérieurement, la vidéo $360^{\circ}$ se différencie de la réalité virtuelle parce qu'elle ne permet pas, entre autres, à l'utilisateur d'interagir ou de modifier les évènements s'y déroulant (Roche et Rolland, 2020). Elle invite toutefois à visionner une scène du monde réel, sous forme d'immersion visuelle (Fuchs, 2017), en ayant la possibilité de voir dans toutes les directions. De manière vulgarisée, la vidéo $360^{\circ}$ "donne tout simplement l'impression d'être dans l'endroit filmé ». La réalisation de telles vidéos nécessite un équipement particulier comprenant principalement une caméra à 6 lentilles qui peut filmer l'entièreté de l'environnement physique utilisé pour une scène. 


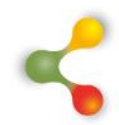

\section{REVUE HYBRIDE DE L'ÉDUCATION}

Le visionnement d'une vidéo de réalité immersive peut se faire de diverses manières. En effet, l'étudiant peut utiliser un casque de réalité virtuelle, mais peut aussi regarder celle-ci sur son ordinateur ou son téléphone intelligent. II utilise alors son curseur pour pouvoir visionner les différents angles de la scène filmée. Dans tous les cas, il peut accorder son attention à certains éléments de la scène en particulier (ex. : se tourner ou déplacer son curseur vers la droite pour mieux voir une intervention dans cette direction).

Les deux enseignants-chercheurs rencontrés ont exploité cette forme de vidéo, mais en contexte pédagogique. Pour ce faire, ils travaillent en collaboration avec différents acteurs et parcourent une panoplie d'étapes, lesquelles sont présentées dans la figure 1.
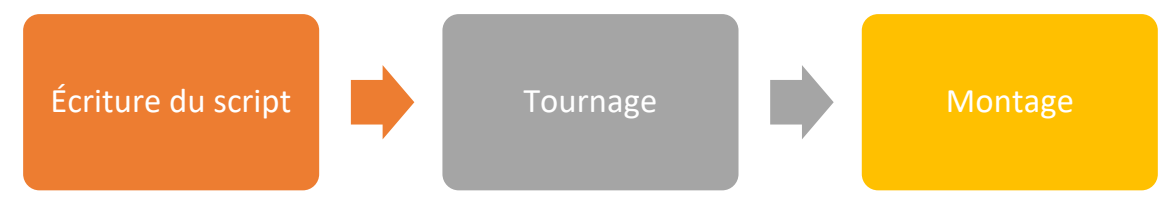

Figure 1 : Étapes de réalisation d'une vidéo $360^{\circ}$

\section{Étape 1 - L'écriture du script}

La première étape demeure d'écrire le script relevant de la scène à piloter. Ce script est réalisé par les experts de contenus, soit les enseignants du programme ciblé. Chaque vidéo qu'ils créent doit représenter une mise en situation en contexte réel liée à un savoir à acquérir ou une compétence à développer (ex. : comment mettre un pansement).

Les experts de contenus prennent soin de rédiger le texte de l'intervenant (ex. : infirmier, policier), des individus avec qui ils interagissent (ex. : patient, citoyen qui enfreint un règlement) et du narrateur (photo 1). Leur objectif est de reproduire le plus familièrement possible les tâches que l'intervenant doit réaliser dans la mise en situation, lesquelles seront décrites de manière plus théorique par le narrateur. Si une vidéo met en scène un infirmier, par exemple, ce dernier réalisera ses tâches comme s'il était dans son milieu naturel de travail (ex. : saluer le patient, demander la date de naissance du patient, prendre ses signes vitaux, etc.). Le narrateur, de son côté, verbalisera les raisons qui justifient chacun de ses gestes en utilisant le vocabulaire lié au domaine d'études (ex. : demander la date de naissance pour s'assurer qu'il s'agit du bon patient). 


\section{REVUE HYBRIDE DE L'ÉDUCATION}

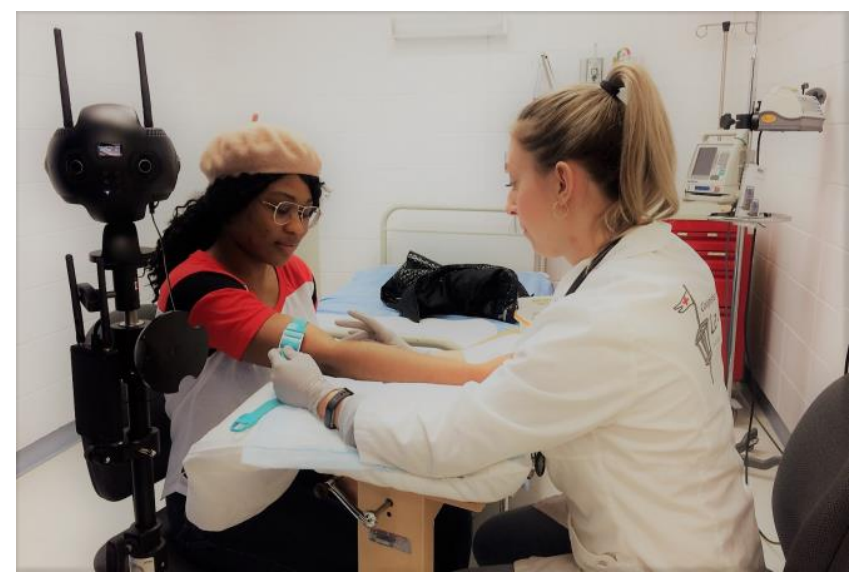

Photo 1 : Deux acteurs interprétant respectivement une patiente (à gauche) et une infirmière (à droite).

Crédit photo : Équipe XR Collège d'Alma

\section{Étape 2 - Le tournage}

Comme ce dispositif est considéré davantage comme un film qu'un tutoriel par les deux enseignants-chercheurs consultés, il demeure nécessaire d'embaucher des acteurs pour interpréter les scènes imaginées et de s'assurer d'une bonne qualité visuelle et sonore lors de l'enregistrement. Pour ce faire, ils collaborent avec des techniciens du programme de technologie sonore proposé dans leur collège. Comme précisé antérieurement, une caméra spécialisée est à cette étape utilisée pour s'assurer de filmer la scène sous tous ses angles (photo 2). 


\section{REVUE HYBRIDE DE L'ÉDUCATION}

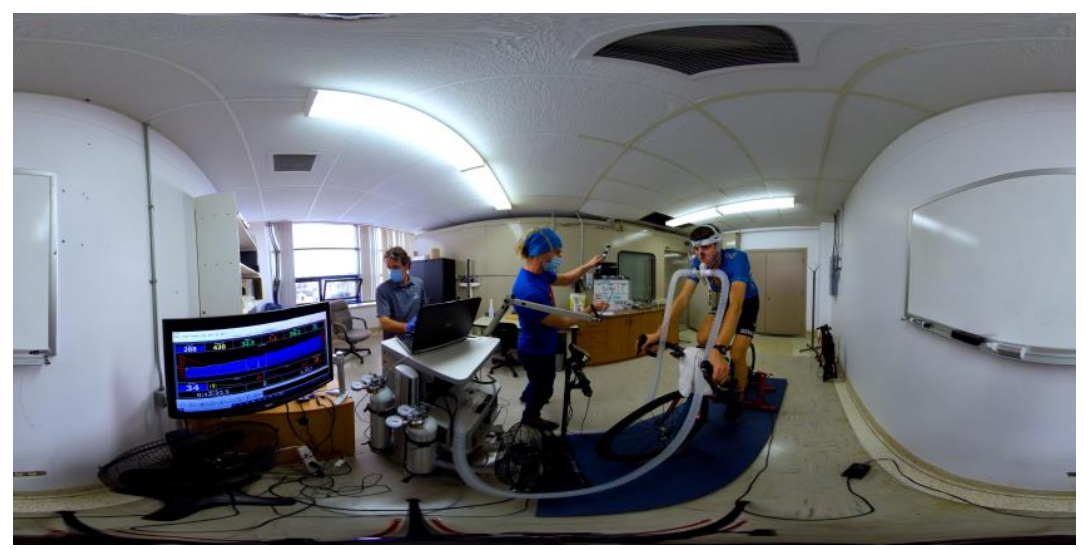

Photo 2 : Image équirectangulaire tirée d'une vidéo en réalité immersive $\mathbf{3 6 0}$ pour le programme de sciences de la nature. Crédit photo : Équipe XR Collège d'Alma ${ }^{1}$

\section{Étape 3 - Le montage}

La dernière étape demeure de réaliser le montage de la vidéo et de la rendre accessible aux étudiants du programme.

\section{Apports et prospectives}

De nombreuses retombées pédagogiques découlant de l'utilisation de la vidéo $360^{\circ}$ sont perçues par les enseignants-chercheurs interrogés. Ils ont d'ailleurs obtenu une subvention de recherche du Programme d'aide à la recherche sur l'enseignement et l'apprentissage (PARÉA) du ministère de l'Éducation et de l'Enseignement supérieur qui vise à évaluer l'incidence de la formation immersive sur l'acquisition de différents savoirs par les étudiants en soins infirmiers. Leurs travaux permettront notamment de vérifier si leurs perceptions des retombées de ce dispositif d'enseignement s'avèrent vraies.

Pour les étudiants, il s'agit évidemment d'une façon efficace de comprendre davantage certaines notions abordées dans leur programme de formation en ayant l'opportunité de visionner autant de fois que désiré le contenu des vidéos. Comme le soulève un des enseignants-chercheurs rencontrés, cette initiative pédagogique est directement liée à la conception universelle de l'apprentissage : «Certains étudiants ont besoin de voir la technique quelques fois, d'autres c'est 15 fois avant d'avoir la maitrise. Les étudiants deviennent autonomes dans leurs apprentissages. " L'outil peut

\footnotetext{
1 Sur la photo se trouvent Léandre Bouchard (cycliste olympique) et Gilles Nédelec (enseignant du Collège d'Alma)
} 


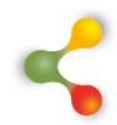

\section{REVUE HYBRIDE DE L'ÉDUCATION}

ainsi être utile pour les étudiants qui ont besoin de plus de temps pour développer certaines de leurs compétences professionnelles.

Pour eux, la vidéo $360^{\circ}$ demeure un outil pédagogique supplémentaire pour consolider les apprentissages des étudiants, en complément avec d'autres approches pédagogiques et l'enseignement régulier. Elle leur offre la possibilité de se familiariser avec certaines techniques ou notions théoriques avant de pouvoir les intégrer et les appliquer concrètement dans leur futur milieu de travail.

Les travaux de ces deux enseignants-chercheurs vont bon train. Ils sont d'avis que les prochaines étapes leur permettront de bonifier leurs techniques de travail liées à l'exploitation de la réalité immersive en contexte pédagogique et de collaborer avec des enseignants de différents programmes, ici et ailleurs, afin de réussir, d'abord et avant tout, à favoriser la réussite des étudiants. 


\section{REVUE HYBRIDE DE L'ÉDUCATION}

\section{Références}

Aguayo, C., Cochrane, T. et Narayan, V. (2017). Key themes in mobile learning: Prospects for learner-generated learning through $A R$ and VR. Australasian Journal of Educational Technology, 33(6), 27-40. https://ajet.org.au/index.php/AJET/article/view/3671/1492

Fuchs, P. (2018). Théorie de la réalité virtuelle les véritables usages. Presses des Mines.

Harrington, C. M., Kavanagh, D. O., Wright Ballester, G., Wright Ballester, A., Dicker, P., Traynor, O., Hill, A. et Tierney, S. (2018). $360^{\circ}$ Operative Videos: A Randomised Cross-Over Study Evaluating Attentiveness and Information Retention. Journal of Surgical Education, 75(4), 993 1000. https://doi.org/10.1016/j.jsurg.2017.10. $\underline{010}$

Roche, L. et Rolland, C. (2020). L'usage de la vidéo $360^{\circ}$ dans la formation des enseignants pour « entrer » virtuellement en classe. Médiations et médiatisations, 4, 27-39. https://revuemediations.teluq.ca/index.php/Distances/article/view/151/91

Stockless, A. (2018). Le numérique en éducation : apprendre en ouvrant les murs de la classe. Médiations et médiatisations, 1(1), 3-5. https://revue-

mediations.teluq.ca/index.php/Distances/article/view/63/31 